\title{
Fibrous Random Materials: From Microstructure to Macroscopic Properties
}

\author{
K. Yazdchi and S. Luding \\ Multi Scale Mechanics (MSM), MESA + Institute for Nanotechnology, Faculty of Engineering Technology, \\ University of Twente, P.O. Box 217, 7500 AE Enschede, The Netherlands
}

\begin{abstract}
Fibrous porous materials are involved in a wide range of applications including composite materials, fuel cells, heat exchangers and (biological)filters. Fluid flow through these materials plays an important role in many engineering applications and processes, such as textiles and paper manufacturing or transport of (under)ground water and pollutants. While most porous materials have complex geometry, some can be seen as two-dimensional particulate/fibrous systems, in which we introduce several microscopic quantities, based on Voronoi and Delaunay tessellations, to characterize their microstructure. In particular, by analyzing the topological properties of Voronoi polygons, we observe a smooth transition from disorder to order, for increasing packing fraction. Using fully resolved finite element (FE) simulations of Newtonian, incompressible fluid flow perpendicular to the fibres, the macroscopic permeability is calculated in creeping flow regimes. The effect of fibre arrangement and local crystalline regions on the macroscopic permeability is discussed and the macroscopic property is linked to the microscopic structural quantities.
\end{abstract}

Keywords: FEM; Delaunay triangulation; Transport properties; Microstructure; Porous media; Permeability PACS: 47.11.Fg; 74.25.F; 61.72.-y; 47.56.+r

\section{INTRODUCTION}

Fluid flows through fibrous materials occur in a class of natural and engineering systems, including filtration, biological interfaces, tissue engineering, fuel cell, fiber reinforcement composite and textile fabric. There has been significant progress during the past decades in our conceptual understanding of the physics of flow and heat/mass transfer in naturally heterogeneous or random porous media [1-6]. However, the relation between such macroscopic properties and the microstructure of such essentially two-dimensional particle systems is an open issue.

The velocity, pressure, and the distribution of fluid flow in this kind of macro-porous medium are the important keys. Because of the important role of permeability, $K$ in porous media, many studies have been devoted to predict its value for low to high porosity by empirical, analytical or numerical models. Well-known models in the granular porous media are (i) lubrication approximation valid for ordered structures at low porosities [7], (ii) the empirical models of Ergun and/or Carman-Kozeny relation valid at moderate porosities [8] and (iii) unit cell approach valid only at high porosities [9]. These models, based on the hydraulic radius model or capillary tube, predict the pressure drop through the porous media. By combining with Darcy's law, one can estimate the permeability from these models.

Despite all these models and attempts, the effect of microstructure (or fibre arrangements) on macroscopic permeability, as a unified relationship, is still unclear. To fill this gap, this paper aims at (i) computationally investigating transverse flow through random fibre arrays in a wide range of porosities, (ii) understanding and characterizing the microstructure, i.e. the ordered and disordered states, using several order parameters and (iii) establishing a unified relationship between macroscopic permeability and the microstructure of the fibrous, 2D granular material.

\section{MICROSTRUCTURAL ANALYSIS}

An important element in understanding of fibrous materials is the description of the local fibre arrangements and the possible correlations between their positions. The classical way for characterizing the structure, like disorder to order transition, is by inspection of its radial distribution function, $g(r)$ which is defined as the probability of finding the centre of a fibre inside an annulus of internal radius, $r$ and thickness $\mathrm{d} r$ [2]. As the crystallization begins to occur at moderate porosities, peaks appear for values of $r$ which correspond to the second (linear) neighbors in a hexagonal lattice in $2 \mathrm{D}$ or a $\mathrm{FCC}$ or $\mathrm{HCP}$ arrangements in $3 \mathrm{D}$. The complete randomness of the fibre distribution on larger scale will assure that $g(r)=1$. However, as pointed out by Rintoul and Torquato [10], this method is unsatisfying for two reasons: on the one hand the absence of clear peaks does not necessarily mean the absence of 
crystallization, and on the other hand it is difficult to determine exactly when the peak appears.

As an alternative, the Voronoi tessellation can be used to study the local and/or global ordering of packings of discs/spheres in 2D/3D. Motivation stems from their variety of applications in studying correlations in packings of spheres, analysis for crystalline solids and super-cooled liquids, the growth of cellular materials and the geometrical analysis of colloidal aggregation and plasma dust crystals [11].

For equal discs, as considered here, given a set of two or more but a finite number of distinct points (generators) in the Euclidean plane, we associate all locations in that space with the closest member(s) of the point set with respect to the Euclidean distance. The result is a tessellation, called Voronoi diagram, of the plane into a set of regions associated with members of the point set, see red lines in Fig. 1. This construction is unique and fills the whole space with convex polygons. In a hexagonally close packed (densest) configuration, i.e. , the Voronoi tessellation consists of regular hexagons. It allows us to define the notion of "neighbor" without ambiguity for any packing fraction: two spheres/discs are neighbor if their Voronoi polyhedra share one face/edge.

The Delaunay triangulation (DT) is the dual graph of the Voronoi diagram. This graph has a node for every Voronoi cell and has an edge between two nodes if the corresponding cells share an edge, see blue lines in Fig. 1. DT cells are always triangles in 2D, and are thus typically smaller than Voronoi cells. The Delaunay tessellation embodies the same information as the Voronoi tessellation. The properties of the latter have been studied before [11], however, it appears that the Voronoi tessellation does not provide information that is directly relevant to the connectivity of pores, which is found to be useful in analyzing the fluid flow in porous media. In the following, to gain further insight into the relative arrangement of the Voronoi cells, their topological correlations have been analyzed at various porosities.

A Monte Carlo (MC) approach was used to generate randomly distributed, non-overlapping fibre/disc arrays, with $N=3000$ particles, in a square domain with length, $L$. Given an initial fibre configuration on a triangular lattice, the $\mathrm{MC}$ procedure perturbs fibre centre locations in randomly chosen directions and magnitudes. The perturbation was rejected if it leads to overlap with a neighboring disk (up to $10^{4}$ perturbations were used in our simulations). With this procedure, we were able to generate various packings at different porosities, $\varepsilon=1-N \pi d^{2} /\left(4 L^{2}\right)$ with $d$ the diameter of fibres, varying from dense/ordered $(\varepsilon=0.3)$ to very dilute/disordered $(\varepsilon=0.95)$ regimes. Similar to Yazdchi et al. [2], a minimal distance, $\Delta_{\min }=\delta_{\min } / d=0.05$ is needed in $2 \mathrm{D}$ to avoid complete blockage. Assigned a virtual diameter $d^{*}=d\left(1+\Delta_{\min }\right)$ to each fiber, leads to the virtual porosity $\varepsilon^{*}=1-(1-$ $\varepsilon)\left(1+\Delta_{\min }\right)^{2}$. While $\varepsilon$ represents the porosity available for the fluid, $\varepsilon^{*}$ (i.e. porosity with artificially enlarged particles) is actually used for packing generation.

The distributions of the cell topologies, i.e. the probability distribution of $n$-sided polygons, $p(n)$ of Voronoi tessellations, generated at various porosities are observed to follow a discretised and truncated Gaussian shape, for more details see [4]. The perfectly ordered structure is manifested by hexagonal cells, i.e. $n=6$ and $p(n)=1$, and disorder/randomness shows up as the presence of cells with other than six sides (topological defects). The increase of disorder in the disc assemblies at high porosities leads to an increase of the topological defect concentration, i.e. a broadening of $p(n)$. In the literature, both the topological defect concentration 1-p(6), and the variance (2nd central moment) of the cell topologies, are used as measures of the degree of disorder. Lemaítre et al. [12] were, to our knowledge, the first to suggest that the equation of state $\mu_{2}=f(p(6))$ could be universal in mosaics. In this sense, all information about topological disorder in these systems is contained in $p(6)$. Astonishingly, Lemaítre's law holds very robustly for most of experimental, numerical, and analytical data [13].

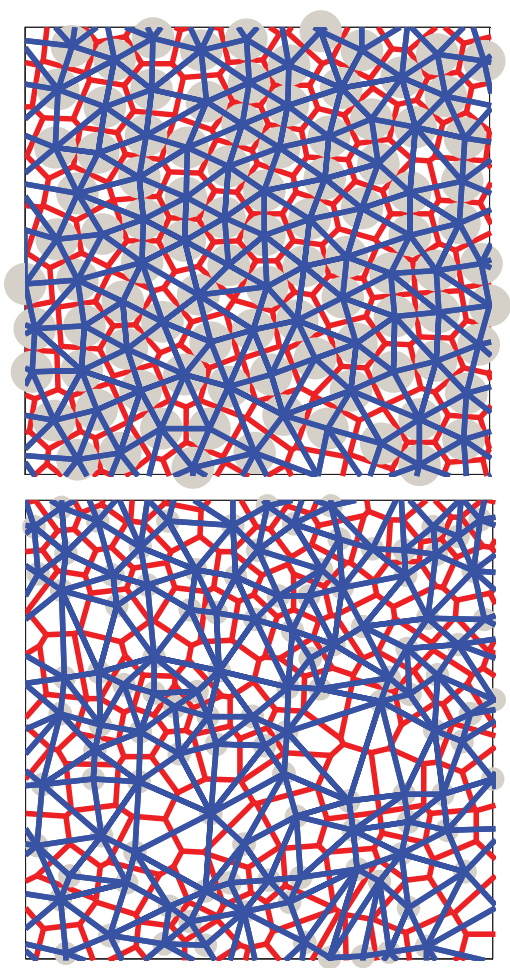

FIGURE 1. Illustration of the Voronoi (red line) and Delaunay (blue lines) tessellations for the center part of a 
system of identical discs at (top) dense, $\varepsilon=0.4$ and (bottom) dilute, $\varepsilon=0.8$, regimes.

Figure 2(a) shows the correlation between $p(6)$ and the topological variance, $\mu_{2}$ for different porosities. In the ordered regime, i.e. $p(6)>0.65$, mainly 5,6 and 7 sided polygons with $p(5) \sim p(7) \sim(1-p(6)) / 2$ occur. By applying the maximum entropy principle [14], we obtain $\mu_{2}=1-p(6)$, which has the trivial virial expansion that corresponds to an ideal gas. By increasing the porosity, i.e. $\varepsilon>0.45$ or $\varepsilon^{*}>0.39$, one enters the disordered regime and $\mu_{2} \sim 1 /\left(2 \pi p^{2}(6)\right)$. Finally, in the limit of vanishing density $(\varepsilon=1)$, the discs are randomly distributed and one has $p(6) \sim 0.3$ and $\mu_{2} \sim 1.78$. This limit is obtained by analyzing the Voronoi polygons generated from $10^{7}$ randomly distributed points [4]. The transition porosity $\varepsilon^{*} \sim 0.39$ can be more clearly determined by plotting the third central moments of the $n$-sided polygon distributions, $\mu_{3}=<(n-<n>)^{3}>$ against porosity, as shown in Fig. 2(b). Note that this value is still far above the random close packing limit $\sim 0.16$, as compared also to the minimum hexagonal lattice porosity $\sim 0.093$, the freezing point $\sim 0.309$ or the melting point $\sim 0.284$ [15].
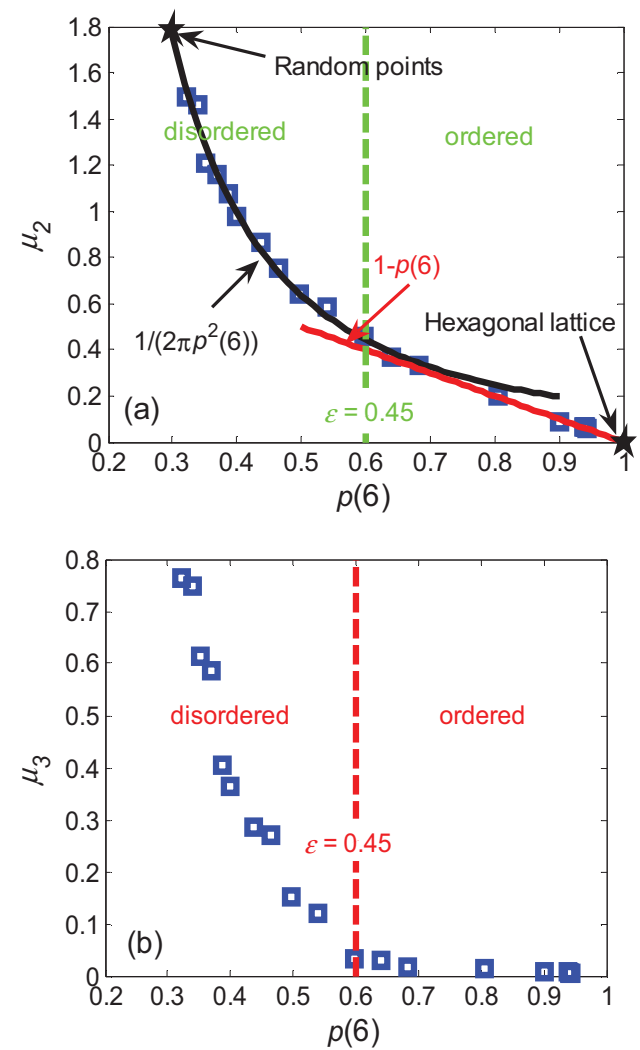

FIGURE 2. (a) The correlation between $p(6)$ and the topological variance $\mu_{2}$ for various structures and porosities.
The analytical theories, represented by solid lines, are calculated by the Maxent method [14, 4]; (b) Variation of the third moment of $n$-sided polygon distributions, $\mu_{3}$ plotted against $p(6)$. The transition from order to disorder occurs at $\varepsilon \sim 0.45\left(\varepsilon^{*} \sim 0.39\right)$. The data are averaged over 10 realizations.

To relate the micro- to macro- properties, in the next section, the macroscopic permeability of these $2 \mathrm{D}$ random structures is calculated numerically, using finite element method (FEM).

\section{DARCY'S LAW AND PERMEABILITY}

Typically, the flow behavior in fibrous media is modeled by Darcy's law, which linearly relates the average velocity in the medium with the pressure gradient, viz.

$$
\vec{U}=\frac{-K \nabla p}{\mu},
$$

where $\vec{U}$ is the superficial velocity, $p$ is the pressure and $\mu$ is the dynamic viscosity of the fluid. In general, $K$ is the second-order permeability tensor, depending on its geometrical parameters including porosity and particles shape, arrangement and/or orientation. For an isotropic porous medium, the permeability becomes a scalar. Darcy's law is valid for the creeping flow in which the Reynolds number is lower than one. In other words, the flow is only influenced by the geometry of pores so the viscous force is important and the inertia force can prove to be negligible.

By employing fully resolved FE simulations of flows through static, regular and random arrays of cylinders, Yazdchi et al. [2] showed that the mean values of the 2 nd nearest neighbor distances of fibres, $\gamma$ (or equivalently the shortest Delaunay triangulation (DT) edges) are nicely correlated with normalized permeability as

$$
\begin{gathered}
K / d^{2}=C \gamma^{2.5} \chi(\gamma), \\
\text { with } \chi(\gamma)=1-0.5 e^{-3 \gamma} \text { and } C \sim 0.2 .
\end{gathered}
$$

Astonishingly, this microstructural model, that resembles lubrication theory, is valid at high and moderate porosities for both ordered and random configurations.

Figure 3 shows the Variation of normalized permeability as function of mean value of shortest DT edges, $\gamma$. The structural transition from disorder to order, indicated by strong increase in $\mu_{3}$, directly affects the macroscopic permeability. In disordered regimes, the permeability data nicely collapse on the 
theoretical power law relation (i.e. $\gamma^{2.5}$ ). However, by appearance the local crystalline regions at $\varepsilon<0.45$, the data start to deviate from the power law. In fact the lubrication theory is only valid for perfectly ordered (hexagonal/square) or disordered (random) configurations with different pre-factor, $C$, in Eq. (2).

Systems that are partially ordered have lower permeability compared to the predicted value of $\gamma^{2.5}$, due to stagnancy of the fluid between fibre aggregates or within crystalline regions of close-by fibres. With decreasing porosity the data deviate from the solid line showing the appearance of ordering in the structure. This deviation is represented by an exponential term in Eq. (2), see red line in Fig. 3.

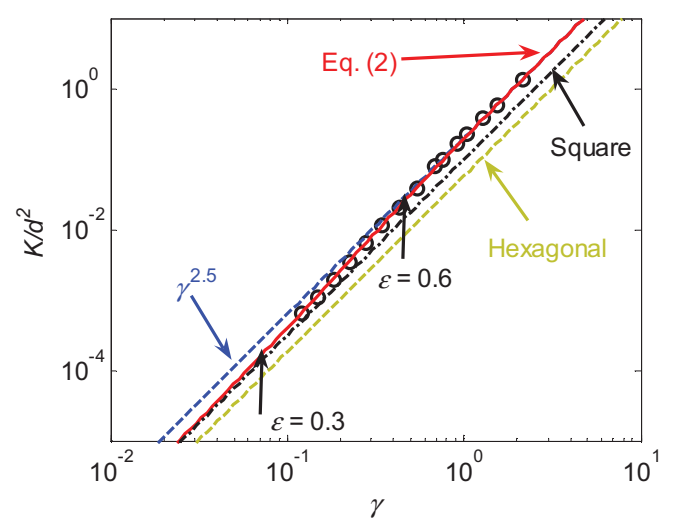

FIGURE 3. Variation of normalized permeability as function of mean value of shortest DT edges, $\gamma$. The black circles show the FE results, averaged over 10 realizations. The results are compared with the square and hexagonal lattice configurations.

\section{SUMMARY AND CONCLUSIONS}

The transverse permeability for creeping flow through unidirectional (dis)ordered arrays of (2D granular) fibers/cylinders has been studied using the finite element method (FEM). Several structural quantities were studied with the goal to characterize the transition, controlled by the effective packing fraction, from disorder to partial order. In this context, the Voronoi and Delaunay diagrams are of interest as they provide information about nearest neighbors, gap distances and other structural properties of fibrous 2D materials. In an ongoing, more general research, Delaunay triangulation has been also used as a contact detection tool and a FE mesh generator in a dense particulate flow model $[16,17]$, at slow, viscous flows. Recently, we observed that the structural transition affects the flow behavior at also inertial (moderate Reynolds numbers) regimes [18].
When the microstructure is characterized by the number of faces of Voronoi polygons and shortest Delaunay triangulation edges or gaps, the 3rd moment of the probability distribution of six-sided Voronoi polygons shows an increase at the transition porosity of $\varepsilon^{*} \sim 0.39$. The numerical experiments suggest a unique, scaling power law relationship between the permeability obtained from fluid flow simulations and the mean value of the shortest Delaunay triangulation gaps. The extension of the present work to 3D structures of (possibly) moving particles still remains a challenge for future study.

\section{ACKNOWLEDGMENTS}

The authors would like to thank S. Srivastava, A. J. C. Ladd, P. Richard, K.W. Desmond and N. Rivier for helpful discussion and acknowledge the financial support of STW through the STW-MuST program, Project Number 10120.

\section{REFERENCES}

1. K. Yazdchi, S. Srivastava, S. Luding, Int. J. Multiphase Flow 37, 956-66 (2011).

2. K. Yazdchi, S. Srivastava and S. Luding, Composites Part A 43, 2007-2020 (2012).

3. K. Yazdchi, "Micro-macro relations for flow through fibrous media", Ph.D. Thesis, University of Twente, 2012.

4. K. Yazdchi and S. Luding, submitted (2012).

5. W.J.B. Grouve, R. Akkerman, R. Loendersloot, S. van den Berg, Int. J Mater Form, Suppl 1, 859-862 (2008).

6. R.B. Bird, W.E. Stewart and E.N. Lightfoot, Transport Phenomena, 2nd edn,. John Wiley \& Sons, 2001.

7. B.R. Gebart, Journal of Composite Materials 26, 1100 33 (1992).

8. P.C. Carman, Transactions of the Institute of Chemical Engineering 15, 150-66 (1937).

9. J.E. Drummond and M.I. Tahir, Int. J. Multiphase Flow 10, 515-40 (1984).

10. M.D. Rintoul and S. Torquato, J. Chem. Phys. 105, 9258- 65 (1996).

11. A. Okabe, B. Boots and K. Sugihara, Spatial Tessellations: Concepts and Applications of Voronoi Diagrams, John Wiley \& Sons, UK, 1992.

12. J. Lemaítre, A. Gervois, J.P. Troadec, N. Rivier, M. Ammi, L. Oger and D. Bideau, Philosophical Magazine Part B 67, 347-62 (1993).

13. J. Lemaítre, J.P. Troadec, A. Gervois and D. Bideau, Europhys. Lett. 14, 77-83 (1991).

14. N. Rivier, NATO Sci. Series 428, 77-93 (1994).

15. B.J. Alder and T.E. Wainwright, Phys. Rev. 127, 359-61 (1962).

16. S. Srivastava, K. Yazdchi, S. Luding, in preparation.

17. K. Yazdchi, S. Srivastava, S. Luding, Powders \& Grains 2013, Sydney, Australia.

18. K. Yazdchi and S. Luding, Chemical Engineering Journal 207, 35-48 (2012). 